\title{
Beschäftigungsfähigkeit, Arbeitsvermögen und Arbeitslosigkeit
}

\author{
Markus Promberger, Ulrich Wenzel, Sabine Pfeiffer, \\ Anne Hacket, Andreas Hirseland
}

\begin{abstract}
Warum sind manche Beschäftigungsverhältnisse stabil, andere nicht? Warum sind die Chancen von Gekündigten oder Arbeitslosen bei der Jobsuche so unterschiedlich? In Zeiten von brüchiger gewordenen Erwerbsbiografien müssen abhängig Erwerbstätige stets erneut beweisen, dass sie für einen Arbeitgeber attraktiv sind. Wem dies nicht gelingt, dem wird häufig mangelnde Beschäftigungsfähigkeit attestiert. Was aber ist eigentlich Beschäftigungsfähigkeit? Der Beitrag zeigt, dass Beschäftigungsfähigkeit mehr ist als die persönlichen, fachlichen und sozialen Fähigkeiten des Einzelnen. Er plädiert für ein umfassenderes Verständnis, das die Nachfragemuster und institutionellen Regelungen des Arbeitsmarktes ebenso umfasst wie die Gelegenheitsstrukturen, die gegeben sein müssen, um Fähigkeiten entfalten und unter Beweis stellen zu können.
\end{abstract}

\section{Einleitung}

Warum finden viele Langzeitarbeitslose keinen passenden Job? Warum gibt es nicht genügend Jobs für alle Langzeitarbeitslosen? Darauf sind im Laufe der Zeit viele Antworten gegeben worden, die sich jeweils mehr oder minder zutreffend mit Teilaspekten des Problems befassen. Die abnehmende Inklusionskraft des Sozialmodells Lohnarbeit (Castel 2002), der technische Fortschritt, die Tertiarisierung (Fourastiè 1969) und der Strukturwandel der Erwerbsarbeit, die institutionell gehemmte Marktpreisbildung für Arbeit (Sargent 1973), die Zwangsläufigkeit der Existenz einer industriellen Reservearmee (Marx 1984) wie auch die Globalisierung (Beck 1999) seien hier beispielhaft als Makroperspektiven genannt. Subjektbezogene Ansätze untersuchen hingegen die Armutsfalle (Gebauer et al. 2003), Lock-In-Effekte arbeitsmarktpolitischer Maßnahmen (z. B. Caliendo et al. 2004), das Wechselverhältnis von Arbeitslosigkeit und Gesundheit (Hollederer/Brand 2006) und - klassisch - die Destrukturierung des Alltags von Arbeitslosen (Jahoda et al. 1975).

Subjektbezogene Erklärungsansätze haben in den letzten Jahren zugenommen. Mit dem vielfach beschriebenen Paradigmenwechsel vom versorgenden zum aktivierenden Wohlfahrtsstaat (zuletzt Dingeldey 2007) ging auch in Deutschland der Ausbau von Institutionen einher, in denen Arbeitslose mit "Aktivierungsexperten“ konfrontiert sind (und vice versa), die sich in der Regel zunächst ein systematisches
Bild über die Marktchancen der von ihnen betreuten Personen machen, bevor sie Beratungs- und Jobangebote, Fördermaßnahmen und gegebenenfalls Sanktionen erteilen. Wenn Langzeitarbeitslose nicht in der Lage sind, Arbeit zu finden, dann kann das - so eine unter Arbeitsmarktexperten gängige Auffassung - an ihrer mangelnden „Beschäftigungsfähigkeit“ liegen. Wer aber hat Sorge zu tragen für die individuelle Beschäftigungsfähigkeit von Erwerbspersonen? Wem obliegt die Verantwortung, für „Selbstmanagement, Selbstmarketing und ... eigene Weiterbildung" (Franken 2006, S. 8), welchen Anteil daran können Betriebe, Beschäftigte, Arbeitsuchende oder die Arbeitsmarktpolitik tragen? Eines scheint klar zu sein: Mit dem heute vieldiskutierten Begriff der Beschäftigungsfähigkeit sollen die Bedingungen des Gelingens von fluiden Erwerbsbiografien besser verstanden und bearbeitet werden können, sind doch die Zeiten durchgängiger Beschäftigung bei einem Arbeitgeber „unwiederbringlich vorbei“, wie es etwa aus den Reihen der Leiharbeitsbranche heißt (Franken 2006, S. 6). Beschäftigungsfähigkeit ist denn auch in aller Munde. Instrumente zu ihrer Messung, Programme und Maßnahmen zu ihrer Steigerung und Evaluationen zu Ausmaß und Hintergründen ihrer Veränderung im Zeitverlauf werden entwickelt und zur Anwendung gebracht. Inmitten dieser vielfältigen Aktivitäten gerät eines jedoch mitunter aus dem Blick: Fast jeder versteht etwas anderes unter Beschäftigungsfähigkeit. Schillernd ist dieser Begriff also zur Genüge, doch worin liegen die hauptsächlichen Streitpunkte in der Debatte?

Eine Durchsicht einschlägiger Studien zeigt, dass die Differenzen bislang vor allem darin bestehen, ob Beschäftigungsfähigkeit allein durch individuelle Merkmale (wie Qualifikation, Motivation usw.) gekenn-

Markus Promberger, Dr. phil., Soziologe, ist
Leiter des Forschungsbereichs „Erwerbslosig-
keit und Teilhabe“ am Institut für Arbeits-
markt- und Berufsforschung (IAB) in
Nürnberg. Arbeitsschwerpunkte: Armuts-,
Arbeits- und Arbeitsmarktforschung.
e-mail: markus.promberger@iab.de
Ulrich Wenzel, Dr. phil., Soziologe, wissen-
schaftlicher Mitarbeiter am Institut für
Arbeitsmarkt- und Berufsforschung (IAB)
in Nürnberg. Arbeitsschwerpunkte: Soziale
Differenzierung und Ungleichheit, qualitative
Methoden, Kommunikations- und Kultur-
soziologie.
e-mail: ulrich.wenze/@iab.de
Sabine Pfeiffer, Dr. phil., Soziologin, wissen-
schaftliche Mitarbeiterin am Institut für So-
zialwissenschaftliche Forschung in München
(ISF). Arbeitsschwerpunkte: sozialwissen-
schaftliche Arbeitsforschung, Informatisie-
rung, Arbeitsvermögen.
e-mail: sabine.pfeiffer@isf-muenchen.de
Anne Hacket, Dipl.-Soziologin, wissenschaft-
liche Mitarbeiterin am Institut für Sozial-
wissenschaftliche Forschung in München (ISF).
Arbeitsschwerpunkte: Arbeitsmarktforschung,
Erwerbsverläufe, soziale Ungleichheit.
e-mail: anne.hacket@isf-muenchen.de
Andreas Hirseland, Dr. rer. pol., Soziologe,
stellv. Leiter des Forschungsbereichs
"Erwerbslosigkeit und Teilhabe“ am Institut
für Arbeitsmarkt- und Berufsforschung (IAB)
in Nürnberg. Arbeitsschwerpunkte: Sozial-
staatsforschung, Diskursanalyse, Über-
schuldungsforschung.
e-mail: andreas.hirseland@iab.de
a

Markus Promberger, Dr. phil., Soziologe, ist Leiter des Forschungsbereichs "Erwerbslosigkeit und Teilhabe" am Institut für Arbeitsmarkt- und Berufsforschung (IAB) in Nürnberg. Arbeitsschwerpunkte: ArmutsArbeits- und Arbeitsmarktforschung. e-mail: markus.promberger@iab.de Ulrich Wenzel, Dr. phil., Soziologe, wissen schaftlicher Mitarbeiter am Institut fü Arbeitsmarkt- und Berufsforschung (IAB) Soziale Methoden, Kommunikations- und Kultursoziologie.

e-mail: ulrich.wenzel@iab.de schaftliche Mitarbeiterin am Institut für Sozialwissenschaftliche Forschung in München rung, Arbeitsvermögen. e-mail: sabine.pfeiffer@isf-muenchen.de Anne Hacket, Dipl.-Soziologin, wissenschaft liche Mitarbeiterin am Institut für Sozial. Arbeitsschwerpunkte: Arbeitsmarktforschung Erwerbsverläufe, soziale Ung/eichheit. ail: anne.hacket@ist-muenchen.de Andreas Hirseland, Dr. rer. pol., Soziologe, stellv. Leiter des Forschungsbereichs "Erwerbslosigkeit und Teilhabe" am Institut staatsforschung, Diskursanalyse, Übere-mail: andreas.hirseland@iab.de 
zeichnet ist oder auch die Marktseite berücksichtigt werden muss. Bei Letzterem würde man zusätzlich auch die Nachfragemuster, die betrieblichen Qualifikationsangebote sowie die institutionellen Rahmenbedingungen untersuchen, wie sie vom Arbeitsrecht oder der Arbeitsförderung gesetzt werden. In diesem Beitrag zeichnen wir die Debatte um den Begriff der Beschäftigungsfähigkeit nach und optieren dafür, bei der Analyse von Beschäftigungsfähigkeit sowohl die individuelle als auch die Markt- und Institutionenseite zu berücksichtigen (Abschnitt 2). Wir meinen jedoch, dass die Diskussion um Beschäftigungsfähigkeit noch einen Schritt weitergehen muss. Bislang wurden diese beiden Komponenten meist so verstanden, als ob sie mehr oder weniger statisch wären: Ein Arbeitnehmer sei mehr oder weniger qualifiziert oder motiviert, ein Betrieb sei mehr oder weniger stark an betrieblicher Weiterbildung interessiert, ein Förderinstrument sei zur Eingliederung von Arbeitslosen mehr oder weniger geeignet. Tatsächlich aber beeinflussen diese Dimensionen die Beschäftigungsfähigkeit nur in vermittelter Weise, nämlich durch ihren Gebrauch in der Praxis. Es kommt eben darauf an, ob die erworbenen Qualifikationen im konkreten Arbeitsumfeld realisiert werden können; ob es einem Betrieb gelingt, die Anforderungen im Umgang mit einem bestimmten technologischen Innovationsschritt wirksam an die Belegschaft zu vermitteln, oder auch, ob Instrumente und Maßnahmen der Arbeitsförderung passgenau dort eingesetzt werden, wo sie benötigt werden und wirken können. In der Praxis ist das längst angekommen, man weiß, dass ein Zeugnis, eine Anweisung oder Vorschrift allein kaum ausreichen, um Entscheidungen treffen zu können: Bewerber werden gerne einmal zum „Probearbeiten" eingeladen, Betriebe und Arbeitsagenturen interessieren sich für „best practice“Beispiele, wenn sie eine neue Aufgabe zu bewältigen haben. Die Arbeitswelt ist in den vergangenen Jahrzehnten unübersichtlicher und beweglicher geworden, und dies muss bei dem Konzept der Beschäftigungsfähigkeit berücksichtigt werden. Da sich aus isoliert messbaren, relativ unveränderlichen Merkmalen von Arbeitnehmern, Betrieben und Institutionen ein vergröbertes, wenn nicht verzerrtes Bild der Beschäftigungsfähigkeit ergibt, müssen die Formen des praktischen Umgangs mit Anforderungen der Arbeitswelt ebenfalls berücksich- tigt werden. Wir schlagen deshalb in diesem Beitrag vor, Beschäftigungsfähigkeit als ein nicht nur interaktiv, sondern auch pragmatisch (im Handeln) zustande kommendes Phänomen $\mathrm{zu}$ betrachten (Abschnitt 3). Dies setzt jedoch voraus, ein erweitertes Verständnis von beschäftigungsrelevanten Kompetenzen zu entwickeln, wie es das Konzept des Arbeitsvermögens bereitstellt (Abschnitt 4). Abschließend werden arbeitsmarktpolitische Implikationen diskutiert, die sich im Anschluss an das hier vorgeschlagene Verständnis pragmatisch-interaktiver Beschäftigungsfähigkeit ergeben (Abschnitt 5).

\section{Beschäftigungsfähigkeit: Individuell oder interaktiv?}

Zwar gibt es einen weitgehend geteilten Minimalkonsens darüber, dass Beschäftigungsfähigkeit das Potenzial von Personen bezeichnen soll, ein Beschäftigungsverhältnis einzugehen, aufrechtzuerhalten oder falls notwendig - durch ein anderes zu ersetzen. Wie sich dieses Potenzial jedoch aufbaut, welche Komponenten es umfasst, ist bislang eine offene Kontroverse, die sich durch unscharfe Ränder und eine häufig additive Begriffsbestimmung auszeichnet.

$\mathrm{Da}$ ist erstens der weite Bereich individueller Merkmale, Fähigkeiten und Orientierungen, der für ein mehr oder minder hohes Maß an Beschäftigungsfähigkeit verantwortlich gemacht wird: Gesundheitlicher Status, berufliche Fertigkeiten, die sich auch in Zeugnissen niederschlagen, Eignungs- und Weiterbildungsprofile, Akzeptanz flexibler Beschäftigungsformen, soziale Kompetenzen wie Team- oder Kritikfähigkeit, soft skills aller Art, Aspekte der sozialen Präsentabilität (wie saubere Kleidung oder höfliches Benehmen) usw. Dieser Bereich wird als individuelle Komponente der Beschäftigungsfähigkeit bezeichnet. Zweitens gibt es auch einen Bereich von institutionellen Regelungen, Nachfragemustern am Arbeitsmarkt und betrieblichen Fördermaßnahmen, auf denen Beschäftigungsfähigkeit aufbauen soll: Gesetzliche Regelungen, die sich auf die Beschäftigungschancen einzelner auswirken (beispielsweise ABM, Regelungen zur Altersteilzeit oder Antidiskriminierungsgesetze), soziale Unterstützungsleistungen, Gesundheitsdienste, selektive Ein- und Ausgliede- rungsmuster in Betrieben, Strategien der Personalauswahl, Fort- und Weiterbildungsangebote usw. Diesen Bereich bezeichnen wir als institutionelle Komponente bzw. Marktkomponente von Beschäftigungsfähigkeit, je nachdem, ob arbeitsmarktrelevante Institutionen oder Nachfragemuster im Vordergrund stehen. Folgt man der einschlägigen Literatur, kann jedes dieser Elemente Beschäftigungsfähigkeit ausmachen - oder auch alle zusammen.

Im EU-Kontext wurde die Förderung von Beschäftigungsfähigkeit vor rund zehn Jahren als Ziel in die Europäische Beschäftigungsstrategie aufgenommen (Gazier 1999a, S. 38f.). In den Leitlinien für 1998 werden unter dieser Überschrift Maßnahmen der Qualifizierung, Beratung und Vermittlung angesprochen, durch die Arbeitslosigkeit bekämpft und die Erwerbsneigung der gesamten Bevölkerung erhöht werden soll. Diese Initiative reihte sich in eine lebendige Debatte um die Begriffsbestimmung von Beschäftigungsfähigkeit ein. Mit dem Job-AQTIV-Gesetz erlangte das Konzept einer „individuellen Beschäftigungsfähigkeit" im Jahr 2002 auch im deutschen Arbeitsförderungsrecht Geltung (\$ 1 SGB III).

Gazier (1999a; 1999b; 2001) unterscheidet drei Wellen der Konzeptualisierung von Beschäftigungsfähigkeit, aus denen sieben unterschiedliche Begriffsdefinitionen hervorgegangen sind. Zum Ende der 1990er Jahre, so Gazier (1999a, S. 43), wurde der Diskurs von Konzepten dominiert, die sich auf den, ,interaktiven “Aspekt des zur Rede stehenden Phänomens konzentrierten. Beschäftigungsfähigkeit resultiert diesem Verständnis zufolge aus dem Wechselspiel zwischen der individuellen Seite einerseits und der institutionellen und Marktseite andererseits. Konzepte mit dieser Ausrichtung wurden für die Evaluation arbeitsmarktpolitischer Instrumente genutzt (Kleffmann et al. 1997; Deeke/ Kruppe 2003, S. 9) und finden sich bis heute vor allem in Studien, die sich auf die problematische Arbeitsmarktlage bestimmter Personengruppen richten. Denn bei solchen Gruppen (beispielsweise Ältere, Behinderte oder Ausländer) ist eine schlechtere Arbeitsmarktperformance nicht selten auch dann zu verzeichnen, wenn die individuellen Voraussetzungen eher gut sind. Forscher, die sich mit solchen möglichen Benachteiligungs- und Diskriminierungseffekten beschäftigen, sehen Beschäftigungsfähigkeit folgerichtig als ein interak- 
tives Phänomen, das nicht nur von den Voraussetzungen des Individuums geprägt ist, sondern ebenso sehr durch institutionelle Regelungen und betriebliche Praktiken, etwa im Bereich Personalrekrutierung und Weiterbildung (Bollérot 2001; Bellmann et al. 2007).

Insgesamt war die Vorrangstellung interaktionaler Konzepte jedoch von kurzer Dauer und hat sich in jüngster Zeit deutlich abgeschwächt. Verstärkt werden nun wieder Begriffsbildungen vertreten, die auf nur eine der beiden „interaktiv“ verbundenen Seiten abstellen, und zwar auf die des Arbeitsangebots. Es handelt sich hierbei um Studien, die - im Sinne des Aktivierungsparadigmas (Leisering 2004; Legnaro 2006; Dingeldey 2007) - davon ausgehen, dass Arbeitsmarktpolitik sich vorrangig auf die quantitative und qualitative Steigerung des Arbeitsangebots konzentrieren müsse, das heißt auf eine hohe Konzessionsbereitschaft, eine schnelle Vermittlung in Arbeit sowie - wo nötig und sinnvoll - Qualifizierung. Wird Beschäftigungsfähigkeit auf diese Weise thematisiert, so geht es um die individuelle Arbeitsneigung und Kompetenzausstattung (Lindsay et al. 2007), gegebenenfalls zusätzlich um Persönlichkeitsmerkmale, die Verfügung über Sozialkapital (Fugate et al. 2004; McArdle et al. 2007) und den Gesundheitszustand. Die Rolle, die institutionelle Regelungen und betriebliche Praktiken bei der Herstellung von individueller Beschäftigungsfähigkeit spielen, wird von Vertretern solcher, auf das Arbeitsangebot zentrierter Konzepte zuweilen zwar anerkannt, nicht aber bei der Operationalisierung berücksichtigt. Bei der aktuellen Evaluation zur SGB II-Experimentierklausel (Vergleich des ARGEund des Optionskommunen-Modells) beispielsweise wird Beschäftigungsfähigkeit als ,individuelles Potenzial zur Aufnahme, Aufrechterhaltung und Ausweitung einer Erwerbstätigkeit" (ZEW et al. 2007, S. 29) definiert und mit einem Bündel von Merkmalen operationalisiert, die sich ausschließlich auf die Seite des Arbeitsangebots beziehen, mithin Merkmale der Person darstellen. ${ }^{1}$ Die institutionellen und nachfrageseitigen Voraussetzungen werden hier nicht der Beschäftigungsfähigkeit zugerechnet, sondern als Governance-Probleme bzw. Marktschwankungen konzeptualisiert, die gesondert zu diskutieren seien.

Merkmale der Person können sich jedoch nur dann in Beschäftigung umsetzen, wenn sie auf korrespondierende Nachfra- gemuster und institutionelle Arrangements stoßen. Und Letztere sind ebenso veränderlich wie die individuellen Merkmale. Individuelle Beschäftigungsfähigkeit kann sich durch Veränderungen auf beiden Seiten verbessern. Personen legen beispielsweise an Beschäftigungsfähigkeit zu, wenn das regionale Arbeitsangebot insgesamt sinkt, weil Betriebe dann tendenziell verstärkt auf weniger qualifizierte oder weniger erfahrene Arbeitskräfte zurückgreifen und parallel dazu betriebliche Qualifizierungsangebote ausdehnen. Ein ähnlicher Effekt kann auch von arbeitsmarktpolitischen Förderinstrumenten ausgehen, beispielsweise von Unterstützungsleistungen in Zeiten der Einarbeitung und Weiterqualifizierung am Arbeitsplatz. ${ }^{2}$

Da die Adressaten der Aktivierungspolitik in der Regel nicht die Arbeitgeber, sondern die Arbeitslosen sind, verwundert es nicht, dass insbesondere die reflektierenden Praktiker der Aktivierung an ihren Klienten Merkmale identifizieren, die deren individuelle Beschäftigungsfähigkeit charakterisieren. Verstärkt wird diese Betroffenenzentrierung möglicherweise auch dadurch, dass zumindest in deutschen Arbeitsämtern oft systematisch zwischen Arbeitslosenvermittlung und Arbeitgeberservices getrennt wird; ein Fallmanager, Arbeitsvermittler, persönlicher Ansprechpartner interagiert vor allem mit seinen arbeitslosen Klienten, die Jobs bekommt er aus der Stellenangebotsdatei, häufig ohne Kontakt mit den Arbeitgebern, die die Jobs anbieten.

In diesem Zusammenhang kommt es leicht zu einem folgenschweren Zuschreibungsprozess. Die Stellenangebote haben fixierten, statischen, in einem Aktensystem festgehaltenen Dokumentencharakter, während die Passung oder Abweichung der arbeitslosen Person zu den Stellenangeboten lebendig und personengebunden erscheint. Was eigentlich ein zweiseitiger Marktprozess ist - die Erwartungen von Arbeitgebern und die Merkmale von Arbeitsuchenden - erscheint in der Tendenz vor allem als persönliche Eigenschaft des Arbeitslosen und wird als Passung oder Abweichung der Person von „objektiven Marktanforderungen“"wahrgenommen.

\section{Beschäftigungsfähigkeit entsteht in der Praxis}

Entgegen der in jüngster Zeit zu verzeichnenden Wiederkehr „einseitiger" Konzeptualisierungen von Beschäftigungsfähigkeit haben wir im voranstehenden Abschnitt argumentiert, dass es die Begriffstradition „interaktiver“ Beschäftigungsfähigkeit ist, die analytisch fruchtbarer und für Evaluationszwecke geeigneter ist. Dieses Argument muss jedoch noch erweitert werden, damit der Begriff den jüngsten Veränderungen der Arbeitswelt gerecht wird. Es ist ja kein Zufall, dass die Rede von der Beschäftigungsfähigkeit sich in den vergangenen Jahrzehnten so stark verbreitet hat; vielmehr drückt sich darin ein Strukturwandel im Verhältnis von Arbeit und Gesellschaft aus. Beschäftigungsfähigkeit ist ein funktionales Äquivalent für jene Strukturierung am Arbeitsmarkt, die in früherer Zeit von verallgemeinernd wirkenden Institutionen des Lebenslaufes und des Arbeitsmarktes ausging. Da sich deren Verbindlichkeit zunehmend abschwächt, sichtbar etwa an der schwindenden Rolle von Beruflichkeit im Arbeitsprozess, gewinnen Beschäftigungsfähigkeit und ihre differenzielle Verteilung eine wachsende Bedeutung. Beschäftigungsfähigkeit trägt damit zunehmend die Last der Vermittlung zwischen verschiedenen Sphären der Gesellschaft, hier insbeson-

\footnotetext{
1 Insbesondere sind dies Qualifikation und Arbeitskompetenzen, Gesundheitszustand, soziale Kompetenzen und bestimmte Persönlichkeitsmerkmale (ZEW et al. 2007, 32ff.).

2 In dieser Dopplung von angebots- und nachfrageseitigen Merkmalen gibt es einen inhaltlichen Zusammenhang zwischen den Begriffen der Beschäftigungsfähigkeit und der Arbeitsmarktferne bzw. -nähe. Auch letzteres Phänomen ist zugleich von individuellen wie institutionellen und betrieblichen Merkmalen, Orientierungen und Handlungsweisen abhängig. Das Konzept der Arbeitsmarktferne bzw. -nähe dient in der Diskussion vorrangig dazu, Mechanismen des Arbeitsmarktausschlusses von Randgruppen zu diskutieren, hat also einen dichotomen Zuschnitt (fern - nah) und befasst sich im Wesentlichen mit Marktzutrittsbedingungen. Beschäftigungsfähigkeit ist demgegenüber ein gradueller Begriff und befasst sich mit weiteren Aspekten der Erwerbsteilhabe, z. B. mit Fragen der Kontinuität von Erwerbsbiografien. Aus diesem Grund konzentrieren wir uns auf Beschäftigungsfähigkeit.
} 


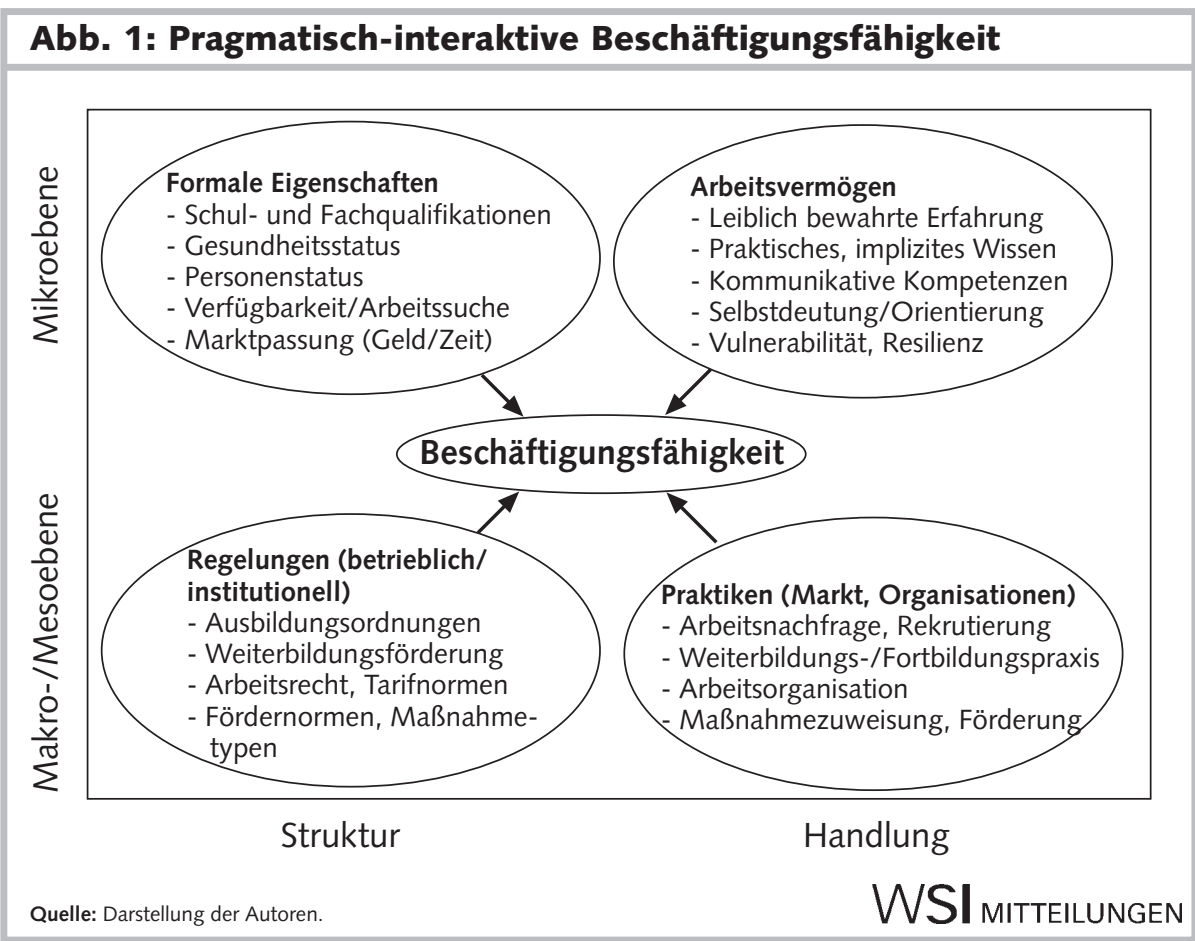

dere zwischen dem Alltagsleben, der Bildungs- und Ausbildungssphäre und der lohnarbeitszentrierten Ökonomie. Aus diesen Gründen kann Beschäftigungsfähigkeit nicht als statisches Merkmal betrachtet werden (sei es nun individuell oder interaktiv), sondern nur als ein dynamisches Phänomen, das im Kontext praktischer Handlungen entsteht und transitorische Gestalt gewinnt. Um relevante gesellschaftliche Prozesse am Arbeitsmarkt zu erfassen, muss der Begriff analytisch geeignet sein, die komplexen Beziehungen zwischen den sich verändernden Bildungsund Erwerbsbiografien einerseits und den sich verändernden institutionellen Regelungen und Nachfragemustern andererseits zu erfassen. Nur so trägt der Begriff der Beschäftigungsfähigkeit zum Verständnis eines sich wandelnden Systems der Erwerbsarbeit bei (ähnlich Bartelheimer 2007).

Im Mittelpunkt des Konzepts „Beschäftigungsfähigkeit" müssen angesichts dieser Wandlungen folglich die fluideren, dynamisierten und partikularer gewordenen Muster des gesellschaftlichen Austauschs zwischen Erwerbstätigen, Unternehmen, Sozialpartnern und staatlichen Instanzen der Arbeitsmarktregulation stehen. Beschäftigungsfähigkeit hat infolgedessen nicht nur eine „interaktive“, sondern auch eine „pragmatische“ Gestalt. Pragmatisch (von griechisch „pragma“, die Handlung) ist Beschäftigungsfähigkeit, weil es nicht

beziehung zwischen einem Beschäftigten und seinem Vorgesetztem und z. B. einem Lehrgangsleiter in tatsächlichen Nutzen überführt werden. Formale Merkmale als Elemente der Beschäftigungsfähigkeit gewinnen also ihre tatsächliche Bedeutung und mögliche Wirkung für die Erwerbsbiografie des Einzelnen erst im Zuge ihres praktischen Gebrauchs. Und dieser Gebrauch, ihr Einsatz, ist von den feststehenden Merkmalen selbst nicht direkt ableitbar.

Insbesondere gilt dies für das Arbeitshandeln selbst, das mehr ist als eine bloße Umsetzung von Erlerntem. Es umfasst stets auch ein Moment von Adaptivität und Kreativität, es generiert somit neues oder verändertes Wissen. Gleiches gilt für das Handeln von Arbeitgebern, die geltende Regelungen oder arbeitsmarktpolitische Förderangebote auf die Erfordernisse der je konkreten Situation anwenden. Erst aus dem Wechselspiel zwischen strukturellen Handlungsvoraussetzungen und deren pragmatischer Aneignung in konkreten Situationen des Erwerbslebens entsteht oder vergeht Beschäftigungsfähigkeit. Aus diesem Grund schlagen wir vor, Beschäftigungsfähigkeit als „pragmatisch-interaktiv“ konstruierte Eigenschaft zu verstehen, also eingebettet in eine zweidimensionale Matrix. In der einen Dimension wird zwischen den beteiligten Ebenen unterschieden, also zwischen institutionellen Regelungen und Betrieben einerseits (Makround Mesoebene) und den individuellen Wissens- und Handlungskompetenzen andererseits (Mikroebene). In der anderen Dimension wird zwischen tendenziell verfestigten und formalisierten Aspekten von Beschäftigungsverhältnissen einerseits (Struktur) und den fluideren, handlungsorientierten und situationsgebundeneren Aspekten andererseits (Handlung) unterschieden. Damit ergibt sich eine Vierfelder-Tafel, die die Komponenten von Beschäftigungsfähigkeit darstellt (Abbildung 1).

Dieser Begriffsvorschlag einer pragmatisch-interaktiven Beschäftigungsfähigkeit erfordert auch eine Neukonzeptualisierung der Subjektseite im Prozess der Arbeitssuche. Bleibt doch die Frage berechtigt, welche Potenziale die Arbeit suchenden Menschen auf den Arbeitsmarkt mitbringen und vor allem: wie sie dieses Potenzial jeweils entwickeln und erhalten. Hierfür bietet sich das Konzept des Arbeitsvermögens an, wie es im Folgenden dargestellt werden soll.

\section{1}

\section{Das Konzept des Arbeitsvermögens}

Was bieten Arbeitslose auf dem Arbeitsmarkt an? Woraus besteht die personenbezogene Seite einer interaktiv zu verstehenden Beschäftigungsfähigkeit? Zunächst sehr allgemein und wie seit Marx (1984, zuerst 1867) oftmals gezeigt, daraus, Arbeitskraft zu sein, das heißt, keine ausreichenden alternativen Einkommensquellen zu haben, sich jedoch physisch, zeitlich, räumlich und sozial so frei bewegen $\mathrm{zu}$ 
können, dass man einer aushäusigen Erwerbsarbeit nachgehen kann. ${ }^{3}$

Doch die Komplexität marktförmiger, arbeitsteiliger Gesellschaften erfordert bekanntermaßen eine ganze Menge weiterer Fähigkeiten, Fertigkeiten und Qualifikationen, die sich keineswegs auf formelle Aspekte beschränken. Was uns zusätzlich besonders interessiert, ist die Ebene des Wissens, des Verhaltens, des Auftretens, der leiblichen, für den historisch-konkreten Arbeitsprozess nützlichen Fähigkeiten und die Einstellung zur Arbeit. Diese Perspektive des Arbeitsvermögens spielte in der Arbeitslosenforschung bislang kaum eine Rolle und wurde in der Arbeitssoziologie lange vernachlässigt, von Ausnahmen abgesehen (Beck-Gernsheim/Ostner 1978; Böhle/Milkau 1988; Böhle 1989). Dieses Arbeitsvermögen ${ }^{4}$ bildet die subjektive, habituelle, leibliche Seite der Arbeit, umfasst Erfahrungs- und inkorporierte Wissensbestände „unterhalb“ der Ebene formaler Zertifikate und generiert so arbeitsbezogene Handlungsfähigkeit (Pfeiffer 2004, S. 137ff.), die es erlaubt, individuelle Befähigung in den Arbeitsprozess hinein zu vermitteln. Die Anwendbarkeit des zertifizierten beruflichen Fachwissens im Rahmen konkreter Arbeitstätigkeiten ist in der Regel Gegenstand von Ausbildungsordnungen, also bereits in die institutionellen Regelungen der Beruflichkeit eingelassen (Kurtz 2005). Mit dem Begriff des Arbeitsvermögens wird ein notwendiges, über diese generellen Anwendungsregeln hinausreichendes Bündel von Wissen und Fähigkeiten beschrieben, das die Verwandlung von Arbeitskraft in Arbeit in je gegebenen, konkreten Situationen ermöglicht (Pfeiffer/Jäger 2006). Ohne entwickeltes Arbeitsvermögen blieben noch so hohe Fachqualifikationen steril, weil ihnen das Moment der Vermittlung in materiale und soziale Umwelten fehlt. Weil Arbeitsvermögen auch diese Momente des Sichin-Beziehung-Setzens umfasst, also Momente von Interaktivität und Kommunikativität, eignet sich dieses Konzept vorzüglich zur Analyse der subjektiven Seite von Beschäftigungsfähigkeit.

Arbeitsvermögen bildet sich aus in (auch leiblicher) Auseinandersetzung mit den materialen (d. h. sowohl stofflichen wie auch organisational und sozial erfahrbaren) Aspekten von anzueignender Welt (Erwerbsarbeit wie alltägliche Lebenswelt) sowie im Umgang mit den hierfür bedeutsamen Mitteln und Gegenständen (Pfeiffer 2004, S. 174ff.). Seine Entwicklung ist also nicht nur Folge der Potenziale des Subjekts, sondern immer auch schon abhängig von den stofflichen, materialen, sozialen Umgebungsbedingungen, auf die das Arbeitsvermögen trifft und an denen es sich ausbildet und aktualisiert. Dies kann von Pünktlichkeit, der Einsicht in die Notwendigkeit der „Fabrikdisziplin“ (Treiber/Steinert 2005) und anderen Formen der psychophysischen Selbstkontrolle sowie des physischtechnischen Gefahrenbewusstseins über habitualisierte Körperhaltungen beim Bewegen schwerer Lasten oder Ausführen repetitiver Arbeiten ${ }^{5}$ reichen. Ebenso umfasst das Arbeitsvermögen die in der älteren Arbeitswissenschaft vorwiegend Frauen zugeschriebene Feinmotorik und Monotonieresistenz (Krell 1984), kommunikative Umgangsformen, Sprachfertigkeiten, Kooperationssitten, Fähigkeiten zur Informationsauswahl und das ,zeitliche Selbstmanagement", fachspezifisches Erfahrungswissen und mehr.

Die vorliegenden Untersuchungen zum Arbeitsvermögen und seinen zentralen Merkmalen, wie Erfahrungswissen und subjektivierendem Arbeitshandeln innerhalb von Erwerbsarbeit (Böhle et al. 2002; Pfeiffer 2004, S. 184f.), zeigen, dass Arbeitsvermögen und die darauf bezogenen Erwartungen des Marktes daran historisch, geschlechtsspezifisch beruflich und sektoral geprägt sind. Welches Arbeitsvermögen in welcher Form marktfähig und damit zu Arbeitskraft wird, verändert sich insbesondere auch im Strukturwandel der Wirtschaft. Dienstleistungsjobs erfordern nicht nur andere Qualifikationen, sondern auch anderes Arbeitsvermögen als Industriearbeit. Standen bei Letzterer noch körperliche Geschicklichkeit, ein Gefühl für technische Abläufe, Materialien und Formen der leiblichen Kooperation im Vordergrund, kommt es mit zunehmender Automatisierung und Informatisierung sowie den neuen, „schlanken" Organisationsformen (lean management) in der Produktion zu einem Wandel des Arbeitsvermögens, das sich den Anforderungen der Dienstleistungsarbeit annähert (Pfeiffer 2007). Zunehmend wichtig werden nun schriftliche wie mündliche Kommunikationskompetenzen, Servicementalität, die Anwendung abstrakter Regeln und Verfahren in konkreten Kontexten, der kompetente Umgang mit „Organisationskulturen " und in jüngerer Zeit insbesondere Fähigkeiten im Umgang mit den automatisierten Systemen der Informations- und Kommunikationstechnologie.
Gerade wenn ältere Arbeitnehmer aus industriellen Produktionsprozessen freigesetzt werden, gelingt es ihnen wegen der geschrumpften Nachfrage nur noch schwer, das in einer langen Biografie erworbene industrielle Arbeitsvermögen auf dem Markt $\mathrm{zu}$ realisieren - und dies ist nicht nur eine Frage formaler Qualifikationen, sondern auch des Habitus und der Erfahrungsbestände. Ohne dass dies hier ausführlicher dargestellt werden kann, ist bereits in dieser Fehlpassung des von Arbeitskräften angebotenen Arbeitsvermögens an die Erwartungen der Arbeitgeber ein Erklärungsbeitrag zur Langzeitarbeitslosigkeit zu vermuten. Bestände und Entwicklungen des Arbeitsvermögens außerhalb von Erwerbsarbeit sind daher von Bedeutung für die Bekämpfung der Arbeitslosigkeit. Zu untersuchen wären hier etwa die Entwicklung und Erosion von Arbeitsvermögen bei Arbeitslosigkeit, deren stabilisierende und destabilisierende lebensweltliche Kontexte sowie die entsprechenden Effekte institutionellen Handelns, sei es in der analytischen Bestandsaufnahme („Profiling“) oder in arbeitsmarktpolitischen Maßnahmen, die die Chancen von Erwerbslosen verbessern sollen.

\section{Zusammenfassung und Konsequenzen für die Arbeits(markt)politik}

Beschäftigungsfähigkeit wird im Gefolge des Aktivierungsparadigmas in jüngerer Zeit vornehmlich auf individuelle Voraussetzungen der Vermittelbarkeit und Arbeitsaufnahme verengt. Demgegenüber erweist sich das Konzept erst in einem

3 Die Definition der "freien Lohnarbeit" geht auf Marx (1984, S. 181ff.) zurück, der sie in historischer Abgrenzung von der feudalen Arbeit formulierte. Doch auch heute noch ist Erwerbsbeteiligung, Arbeitskraft sein, höchst voraussetzungsreich, wie z. B. die Arbeitsmarktprobleme von Alleinerziehenden, Eltern kleiner Kinder, gesundheitlich eingeschränkten Menschen zeigen.

4 „Unter Arbeitskraft oder Arbeitsvermögen verstehen wir den Inbegriff der physischen und geistigen Fähigkeiten, die in der Leiblichkeit, der lebendigen Persönlichkeit eines Menschen existieren und die er in Bewegung setzt, sooft er Gebrauchswerte irgendeiner Art produziert." (Marx 1984, S. 181).

5 Vgl. etwa den Begriff der "Übung“ bei Weber (1909). 
pragmatisch-interaktiven Verständnis als tragfähig, das vier Komponenten berücksichtigt: Erstens die institutionellen Regelungen zu Bildung, Berufen und Arbeit, zweitens die Anforderungen und konkreten Praktiken bei Arbeitgebern und arbeitsmarktpolitischen Organisationen, drittens die formalen Merkmale der Personen und viertens eine subjektgebundene Komponente, die oben näher beleuchtet wurde. Diese Komponente reicht von expliziten Fach- und Schlüsselfähigkeiten und impliziten Erfahrungsbeständen bis hin zu basalen körperlichen und psychosozialen Kompetenzen, habitualisierten Praktiken und Techniken, vom Gefahrensinn über Selbstkontrolle, Fähigkeit zur Alltagsorganisation und zur umfeldadäquaten Selbstpräsentation und Kommunikation. Gemeinsam ist diesen Fertigkeiten und Kompetenzen, dass sie sich erst im Arbeitshandeln realisieren, dass sie kein theoretisches, sondern ein praktisches Wissen und Können darstellen. Im Anschluss an arbeitssoziologische Konzepte wird für diese vierte Komponente der Terminus „Arbeitsvermögen" benutzt. Denn das Arbeitsvermögen ist - wie wir meinen - ein Forschungsgegenstand eigenen Zuschnitts und von hoher Bedeutung, der analytisch und begrifflich zunächst von den anderen Komponenten der Beschäftigungsfähigkeit zu trennen ist.

Den Hintergrund für das Forschungsinteresse am Arbeitsvermögen (Hirseland et al.2007) bildet die arbeitspolitisch höchst relevante Frage, wie Langzeitarbeitslosigkeit, sozioökonomischer Strukturwandel und individuelle Fähigkeiten zusammenwirken. Bislang wurde dieses Problem - wie wir meinen: irreführend - unter dem Label der Beschäftigungsfähigkeit, verstanden als Bündel von Merkmalen, diskutiert. Hier sehen wir eine Tendenz zur Verengung gesellschaftlicher Probleme des Arbeitsmarkts auf die relativ feststehenden Merkmale Arbeit suchender Personen. Unser Plädoyer für ein Konzept der pragmatisch- interaktiven Beschäftigungsfähigkeit zielt demgegenüber darauf $a b$, die Bedingungen gelingender oder misslingender Erwerbsintegration in systematischer Form aufzufächern und dabei zu berücksichtigen, dass es stets Prozesse sozialer Praxis sind, in denen sich die Beziehungen zwischen Personen, Unternehmen und Organisationen der Arbeitsmarktpolitik (und benachbarter Politikfelder) vollziehen (pragmatische Dimension). Nur so kann der zunehmenden Fluidität der Arbeitswelt und der wachsenden Entkopplung der ehedem engen Bindungen zwischen Alltagsleben, Bildungsund Ausbildungswesen und der lohnarbeitszentrierten Erwerbsarbeit Rechnung getragen werden. Die hier vorgestellten Überlegungen dienen zunächst dazu, eine Heuristik für die Analyse von arbeitsmarktlichen Integrations- und Ausschlussbedingungen zu entwickeln, zielen aber mittelfristig auf einen Vorschlag zur Operationalisierung pragmatisch-interaktiver Beschäftigungsfähigkeit ab.

Vor diesem Hintergrund lassen sich zwei - wie wir meinen - überraschende und arbeitspolitisch höchst relevante Forschungsfragen oder Hypothesen formulieren: Erstens liegen bei einem Gutteil der Langzeitarbeitslosen Typen des Arbeitsvermögens vor, die durch industrielle Qualifikationen, Erfahrungen und einen ebensolchen Habitus geprägt und in eine wissensbasierte Dienstleistungsökonomie nur sehr eingeschränkt transferierbar ${ }^{6}$ sind - was viele Arbeitsmarktprobleme dieser Gruppe erklären könnte. Zweitens besteht die Frage, ob und wie Arbeitsvermögen im Verlauf der Arbeitslosigkeit erodiert, ob diese Erosion nur auf formal-fachliche Wissensbestände beschränkt bliebe oder die stillschweigenden, teils habituellen Komponenten des Arbeitsvermögens mit einbezöge, und unter welchen subjektbezogen-biografischen Bedingungen dieser Verfall gegebenenfalls aufgehalten werden könnte. Überdies ist mit der hier vorgeschlagenen Neukonzeption der Beschäfti- gungsfähigkeit ein arbeitspolitischer Richtungswechsel verbunden. Er liegt nicht zuletzt darin, die mit einem verengten Begriff der Beschäftigungsfähigkeit einhergehenden latenten Schuldzuschreibungen an die Arbeitslosen aufzugeben und stattdessen ihr Arbeitsvermögen, mitsamt der informellen Kompetenzen, deren Pflege, Ausbau und Adaption, an die geänderten sozioökonomischen Rahmenbedingungen einer wissensbasierten Dienstleistungsgesellschaft in den Mittelpunkt der aktiven Arbeitsmarkpolitik zu stellen.

Denn schließlich sollen Beratung, Betreuung und Förderung von Arbeitslosen und Hilfebedürftigen darauf abzielen, verschüttete und ungenutzte Potenziale für die Erwerbsintegration und eigenverantwortliche Lebensführung zu heben. So verlangt es zumindest das Aktivierungsparadigma. Um diesen Schritt wirklich zu gehen, sollte der Blick der damit befassten Institutionen sich offensichtlich auch und gerade auf die nicht-standardisierten und nicht-zertifizierten Komponenten der Arbeitskraft richten, also auf das lebendige Arbeitsvermögen, die Formen des praktischen, impliziten Wissens und der Erfahrung. Diese Ebene der individuellen Kompetenzen zu berücksichtigen, sie gegebenenfalls zu fördern und in den Vermittlungsprozess einzubringen, könnte sich positiv auf die individuelle Beschäftigungsfähigkeit auswirken, ebenso wie - und komplementär hierzu - eine Verbesserung der Anpassungsfähigkeit und Aufnahmefähigkeit der betrieblichen Arbeitsnachfrage und Weiterbildungspraxis. 
Bartelheimer, P. (2007): Politik der Teilhabe. Ein soziologischer Beipackzettel. Fachforum Analysen und Kommentare der Friedrich EbertStiftung 1

Beck, U. (1999): Schöne neue Arbeitswelt, Frankfurt Beck-Gernsheim, E./Ostner, I. (1978): Frauen verändern - Berufe nicht? Ein theoretischer Ansatz zur Problematik von „Frau und Beruf“, Soziale Welt 29, S. 257-287

Bellmann, L./Kistler, E./Wahse, J. (2007): Demographischer Wandel: Betriebe müssen sich auf alternde Belegschaften einstellen, IAB-Kurzbericht 21, Nürnberg

Böhle, F. (1989): Körper und Wissen - Veränderungen in der sozio-kulturellen Bedeutung körperlicher Arbeit, Soziale Welt 4, S. 497-512

Böhle, F./Bolte, A./Drexel, I./Dunkel, W./Pfeiffer, S. (2002): Umbrüche im gesellschaftlichen Umgang mit Erfahrungswissen. Theoretische Konzepte, empirische Befunde, Perspektiven der Forschung, ISF München Forschungsberichte, München

Böhle, F./Milkau B. (1988): Vom Handrad zum Bildschirm: Eine Untersuchung zur sinnlichen Erfahrung im Arbeitsprozess, Frankfurt am Main Bollérot, P. (2001): Two actors in employability: The employer and the worker, in: Weinert, P./Baukens, M./Bollérot, P./Pineschi-Gapénne, M./ Walwei, U. (Hrsg.): Employability: From Theory to Practice, International Social Security Series, Bd. 7, New Brunswick, S. 51-90

Caliendo, M./Hujer, R./Thomsen, St. L. (2004): Evaluation der Eingliederungseffekte von Arbeitsbeschaffungsmaßnahmen in reguläre Beschäftigung für Teilnehmer in Deutschland, Zeitschrift für Arbeitsmarktforschung 37, S. 211-237

Castel, R. (2002): Die Metamorphosen der Sozialen Frage, Frankfurt Deeke, A./Kruppe, Th. (2003): Beschäftigungsfähigkeit als Evaluationsmaßstab? Inhaltliche und methodische Aspekte der Wirkungsanalyse beruflicher Weiterbildung im Rahmen des ESF-BA-Programms, IAB-Werkstattbericht 1, Nürnberg

Dingeldey, I. (2007): Wohlfahrtsstaatlicher Wandel zwischen „Arbeitszwang " und "Befähigung ", Berliner Journal für Soziologie 17, S. 189-209 Fagan, C./Halpin, B./O'Reilly, J. (2004),"The Dynamics of Service Sector Employment in Germany and the UK - A Comparison Using Household Panel Data“, in: Schmid, G./Gangl, M./Kupka, P. (Hrsg.): Arbeitsmarktpolitik und Strukturwandel: Empirische Analysen. Beiträge zur Arbeitsmarkt- und Berufsforschung 286, Nürnberg, S. 247-270

Fourastié, J. (1969): Die Große Hoffnung des XX. Jahrhunderts, Köln Franken, H. (2006): Eröffnung, in: Brömser, H.-P./Egle, F. (Hrsg.): Employability. Management für den Arbeitsmarkt der Zukunft. Herausforderungen und Chancen durch Integration, Bildung und Gesundheitsvorsorge am Arbeitsplatz, Fachtagung, 26./27. 10., Heidelberg

Fugate, M./Kinicki, A. J./Ashforth, B. E. (2004): Employability: A psychosocial construct, its dimensions, and applications, Journal of Vocational Behavior 65, S. 14-38

Gazier, B. (1999a): Beschäftigungsfähigkeit: Konzepte und politische Maßnahmen, inforMISEP 67/68, S. 38-51

Gazier, B. (Hrsg.) (1999b): Employability, Concepts and Policies, Bericht im Auftrag der Europäischen Kommission GD V, Berlin

Gazier, B. (2001): Employability - the complexity of a policy notion, in: Weinert, P./Baukens, M./Bollerot, P./Pineschi-Gapenne, M./Walwei, U. (Hrsg.): Employability: From Theory to Practice, International Social Security Series, Bd. 7. New Brunswick, S. 3-23
Gebauer, R./Petschauer, H./Vobruba, G. (2003): Wer sitzt in der Armutsfalle? Selbstbehauptung zwischen Sozialhilfe und Arbeitsmarkt, Berlin Hirseland, A./Promberger, M./Wenzel, U. (2007): Armutsdynamik und Arbeitsmarkt. Qualitative Beobachtungen und Befragungen im Feld von Arbeitsmarkt und sozialer Sicherung, in: Promberger, M. (Hrsg.): Neue Daten für die Sozialstaatsforschung, IAB-Forschungsbericht 12, Nürnberg Hollederer, A./Brand, H. (Hrsg.) (2006): Arbeitslosigkeit, Gesundheit und Krankheit, Handbuch Gesundheitswissenschaften, Bern

Jahoda, M./Lazarsfeld, P. F./Zeisel, H. (1975): Die Arbeitslosen von Marienthal. Ein soziographischer Versuch über die Wirkungen langdauernder Arbeitslosigkeit, Frankfurt am Main

Kleffmann, A./Weinmann, S./Föhres, F./Müller, B. (1997): Melba. Psychologische Merkmalprofile zur Eingliederung Behinderter in Arbeit, Projektbericht, Siegen

Krell, G. (1984): Das Bild der Frau in der Arbeitswissenschaft, Frankfurt Kurtz, Th. (2005): Die Berufsform der Gesellschaft, Weilerswist Leisering, L. (2004): Paradigmen sozialer Gerechtigkeit. Normative Diskurse im Umbau des Sozialstaats, in: Liebig, St./Lengfeld, H./Mau, St. (Hrsg.): Verteilungsprobleme und Gerechtigkeit in modernen Gesellschaften, Frankfurt am Main, S. 29-68

Lindsay, C./McQuaid, R. W./Dutton, M. (2007): New approaches to employability in the UK: Combining "Human capital development" and "work first" strategies?, Journal of Social Policy 36, S. 539-560 Marx, K. (1984): Das Kapital. Zur Kritik der politischen Ökonomie, (=MEW 23), Berlin

McArdle, S./Waters, L./Briskoe, J. P./Hall, D. T. (2007): Employability during unemployment: Adaptability, career identity and human and social capital, Journal of Vocational Behavior 71, S. 247-264

Pfeiffer, S. (2004): Arbeitsvermögen. Ein Schlüssel zur Analyse (reflexiver) Informatisierung, Wiesbaden

Pfeiffer, S. (2007): Montage und Erfahrung. Warum ganzheitliche Produktionssysteme menschliches Arbeitsvermögen brauchen, München Pfeiffer, S./ Jäger, W. (2006): Ende des Elends: Marxsche Reformulierung, handlungstheoretischer Beitrag und dialektische Reanimation der Arbeitsund Industriesoziologie, Soziologie 35, S. 7-25

Sargent, Th. (1973): Rational Expectations, the Real Rate of Interest, and the Natural Rate of Unemployment, Brookings Papers on Economic Activity 2/2, S. 429-472

Treiber, H./Steinert, H. (2005): Die Fabrikation des zuverlässigen Menschen, Münster

Weber, M. (1909): Zur Psychophysik der industriellen Arbeit, in: Jaffé, E./Sombart, W./Weber, M. (Hrsg.): Archiv für Sozialwissenschaft und Sozialpolitik, Bd. 12 und 13, Tübingen

ZEW/IAQ/TNS Emnid (2007): Erster Bericht zur Evaluation der Experimentierklausel nach § 6c SGB II - Vergleichende Evaluation des arbeitsmarktpolitischen Erfolgs der Modelle der Aufgabenwahrnehmung "Optierende Kommune" und "Arbeitsgemeinschaft". Untersuchungsfeld 3: "Wirkungs- und Effizienzanalyse“. Download am 14.12.2007 unter http://www.bmas.de/coremedia/generator/22396/property=pdf/f362_ forschungsbericht.pdf 